Mens

revue d'histoire intellectuelle de l'Amérique française

\title{
Le renard ayant la queue coupée ou La luxuriance des études groulxiennes (1999-2003)
}

\section{Pierre Trépanier}

Volume 4, numéro 2, printemps 2004

URI : https://id.erudit.org/iderudit/1024598ar

DOI : https://doi.org/10.7202/1024598ar

Aller au sommaire du numéro

Éditeur(s)

Centre de recherche en civilisation canadienne-française

ISSN

1492-8647 (imprimé)

1927-9299 (numérique)

Découvrir la revue

Citer ce compte rendu

Trépanier, P. (2004). Compte rendu de [Le renard ayant la queue coupée ou La luxuriance des études groulxiennes (1999-2003)]. Mens, 4(2), 273-307.

https://doi.org/10.7202/1024598ar 


\section{LE RENARD AYANT LA QUEUE COUPÉE OU}

LA LUXURIANCE DES ÉTUDES GROULXIENNES

(1999-2003)

Pierre Trépanier Département d'histoire Université de Montréal

Nul besoin d'être visionnaire pour constater un certain chaos, ni philosophe pour en déduire que l'acte de Genèse se perpétue : toute l'affaire était d'en vivre, jusqu'à la vie mentale.

$O$ vanité des trop grands mots! qui me valent cette punition ce soir: vingt places, vingt chaises vides, et bien que je sois assis sur la mienne, le vide pour moi de leurs absences...'

On a peine à croire que la faveur de Lionel Groulx auprès des chercheurs relève exclusivement de la science pure et qu'elle n'ait rien à voir avec l'impasse où se débat une certaine intelligentsia, sinon la nation elle-même. Quoi qu'il en soit, l'abondante moisson groulxienne réjouit. Cette toute récente historiographie - au sens large - incline tantôt vers la fonction de connaissance, tantôt vers la fonction pratique ; elle s'efforce soit de sonder un univers tel qu'en lui-même, 
soit d'en mobiliser la critique au service des débats de l'heure et des causes actuelles. En fait, la position occupée se situe quelque part entre ces deux pôles, selon l'auteur et selon le sujet. L'extraordinaire floraison des études groulxiennes, qui n'échappe à personne, a été préparée de longue main. Sans diminuer le mérite de ceux dont l'espace me contraint à taire les noms, je voudrais saluer l'apport irremplaçable de Juliette Lalonde-Rémillard, collaboratrice compétente de son oncle, gardienne fidèle de ses archives et de son souvenir. Elle lui ressemble d'ailleurs à bien des égards : puissance de travail, discipline, esprit d'indépendance, franc-parler, chaleur humaine, humour. Nul n'a fait plus qu'elle ni pendant plus longtemps pour la connaissance de Groulx. Des générations de chercheurs ont bénéficié de son aide experte. Viennent ensuite, dans mon petit panthéon, le père Benoît Lacroix et Giselle Huot, qui ont lancé le projet d'édition critique de l'œuvre de Groulx ${ }^{2}$. Je m'en voudrais d'oublier Stéphane Stapinsky, brillant, cultivé, érudit, esprit très fin et très pénétrant, qui a animé les défunts Cabiers d'bistoire du Québec au $X X^{e}$ siècle et prête maintenant son talent à l'encyclopédie en ligne de l'Agora.

Depuis 1999, de solides travaux ont contribué à mieux situer Groulx par rapport à ses amis et à ses adversaires. Sans énumérer les articles qui en ont été tirés, je mentionnerai les mémoires de Stéphane Pigeon sur la critique de la Révolution tranquille par Groulx (1956-1967), de Dominique Foisy-Geoffroy sur Esdras Minville (1923-1939) — étude dont la publication est impatiemment attendue —, d'Yves Bégin sur l'antinationaliste Jean-Charles Harvey ${ }^{3}$, ainsi que les thèses de doctorat de Xavier Gélinas et de Pascale Ryan.

La thèse malheureusement encore inédite de Xavier Gélinas, La droite intellectuelle québécoise et la Révolution tranquille (1956-1966), sans porter principalement sur Lionel Groulx, 
offre une contextualisation indispensable à sa connaissance 4 . L'auteur montre qu'une effervescence intellectuelle est aussi observable à droite pendant ces années. Il reconstitue les réseaux de droite depuis la Seconde Guerre mondiale jusqu'au milieu de la décennie 1960, en insistant sur les intellectuels, leurs lieux de concertation, leurs revues et journaux ainsi que leurs modes d'intervention dans l'espace public. Il offre surtout une analyse nuancée de leurs idéologies. Il éclaire et explique leur déroute. Grâce à ce travail exemplaire, Groulx cesse d'être un penseur solitaire pour devenir un intellectuel. Car si l'intellectuel a besoin d'un public pour exister, il ne peut pas non plus se passer du dialogue ou de la guerre des mots avec les autres intellectuels, ceux de sa famille spirituelle et ceux des camps adverses. Pour la première fois, on trouve réunies, en une synthèse documentée et crédible, écrite avec finesse et élégance, les composantes d'une histoire qui jusque-là gisait en quelque sorte en pièces détachées. Le lecteur se laisse volontiers instruire par un guide sûr, bien au fait des droites françaises et en mesure d'établir des liens utiles comme de proposer des interprétations fécondes. L'érudition de l'auteur, appuyée sur une longue familiarisation, l'autorise à faire des mises au point bienvenues. Il faut souligner cette connaissance intime, fruit d'une longue pratique des auteurs de droite et de leurs organes. Donc, pas de jugements à l'emporte-pièce, appuyés sur une bibliographie sommaire et généralement hostile.

Des intellectuels dans la Cité. La Ligue d'action nationale, 1933-1960, de Pascale Ryan, propose, au lieu d'une autre analyse de l'idéologie d'une revue nationaliste souvent scrutée, l'étude du groupe d'intellectuels qui l'ont faite, des figures de l'intellectuel qu'ils représentent à travers les générations, des modèles d'engagement qu'ils adoptent, des stratégies et des réseaux à travers lesquels ils se constituent en ca- 
tégorie sociale spécifique ${ }^{5}$. À cette fin, l'auteur déploie les ressources de la prosopographie pour situer socialement, culturellement et politiquement le groupe observé. Sa méthode permet d'atteindre des strates intermédiaires d'intellectuels, d'étendre l'analyse au-delà des vedettes, tels Groulx, Minville, Laurendeau ou Angers. L'étude porte moins sur des intellectuels dans la singularité de leur parcours que sur un groupe d'intellectuels, une collectivité, dont elle s'applique à cerner les contours, l'évolution et la dynamique. Le propos de l'auteur s'accompagne d'une utile réflexion sur l'intellectuel, bien enracinée dans l'historiographie internationale. Au fil des pages néanmoins, la biographie collective et l'étude des réseaux, intention première de la thèse, s'effacent un peu devant l'analyse idéologique, et les personnages centraux, dont Groulx évidemment, retrouvent leur rôle habituel. La contribution de Pascale Ryan mérite d'être signalée. Son rapprochement avec celle de Gélinas ne peut qu'enrichir l'historiographie du groulxisme. Les rayons des librairies sont encombrés d'essais troussés de deux ou trois idées approximatives arrosées de quelques jets de bile. Voilà une recherche qui a demandé plusieurs années d'efforts patients. Ne devrait-elle pas au moins être condensée en un livre de 150 ou 175 pages?

Marie-Pier Luneau renouvelle aussi le sujet, pour reprendre une formule consacrée, mais elle y réussit plus complètement que Pascale Ryan ${ }^{6}$. Rompant avec l'analyse textuelle de l'œuvre et adoptant un point de vue externe, elle montre comment Groulx a construit sa figure d'auteur et a assuré le rayonnement de son travail d'écrivain. Malgré ses dénégations, il s'est efforcé de mythifier son personnage. Rouerie de paysan, ruse de renard - l'un de ses pseudonymes n'était-il pas «Un renard qui tient à sa queue »? —, surtout stratégie d'homme du succès, qui fait alliance avec le public 
plutôt que de miser sur les notabilités et les institutions du champ littéraire, ce qui est la stratégie de l'homme de la réussite. Qu'on ne dise pas que c'est là un à-côté : Groulx a trop désiré jouer un rôle d'intellectuel, il s'est trop attardé à sa table de travail, il a trop exercé son style, trop aspiré à la reconnaissance littéraire pour qu'on minimise cette part de sa vie. Mi-conscientes, mi-inconscientes, ces longues manœuvres se révèlent d'une cohérence remarquable. Elles sont à considérer dans l'explication de la stature qu'atteint le petit paysan de Vaudreuil, au même titre que la valeur intrinsèque de son œuvre, sa sensibilité aux attentes du public, la réponse qu'il offre aux requêtes de sa nation ou sa foi en la Providence. Car Groulx croyait en sa mission. Comment faire partager cette conviction sans s'attirer des regards amusés, condescendants ou réprobateurs ? De texte en texte, il tisse autour de sa personne le mythe du berger. Il le fait en toute bonne conscience. Le providentialiste sait que la Providence veut avoir besoin de collaborateurs. Aide-toi, le Ciel t'aidera, dit le proverbe. L'auteur affirme que les propos de Gérard Bouchard, dans Les deux chanoines corroborent les siens (p. 14). C'est confondre l'accessoire (le double discours) avec l'essentiel (un persévérant dessein, qui ordonne toute une carrière). L'ouvrage de Marie-Pier Luneau, rigoureux, parfois trop systématique, enlevé, amusant même constitue une contribution originale aux études groulxiennes.

L'un des aspects négligés par les études groulxiennes est l'évolution des idées de Groulx concernant la diaspora et des rapports qu'il entretient avec les divers groupes qui la composent. Dans son mémoire de maitrise et dans ses articles, Damien-Claude Bélanger aborde le cas de la FrancoAméricanie à partir d'une information abondante et sûre ${ }^{7}$. Il examine comment Groulx intègre dans sa construction nationale le cas franco-américain. Chez Groulx, l'opération est fa- 
cilitée du fait que sa conception de la nation est culturelle et spirituelle. La langue y reste subordonnée à la foi. Les programmes de survivance franco-américaine que Groulx élabore successivement portent la marque à la fois des inflexions de sa doctrine relative au Québec et des circonstances de plus en plus problématiques que vit la Franco-Américanie du point de vue de la fidélité nationale. Bélanger insiste à bon droit sur le ruralisme de Groulx tout en le situant par rapport au ruralisme nord-américain. Il suggère de reprendre toute cette question. Il a raison. Sur la base d'un seul texte de Groulx, il est vrai, Harold Bérubé soutient, pour sa part, que le penseur traditionaliste a voulu - au moins une fois - faire de la ville le cœur de la nation, la matrice de la civilisation, en donnant " au cadre urbain moderne un rôle en continuité avec les valeurs et les événements du passé » (pp. 24-25) ${ }^{8}$. Un beau débat en perspective.

Dans Lionel Groulx, les minorités fransaises et la construction de l'identité canadienne-française, thèse importante qu'il faut publier à tout prix, Michel Bock se penche, pour sa part, sur les minorités en territoire canadien et, en particulier, sur les Franco-Ontariens ${ }^{9}$. L'auteur démontre de façon convaincante que la conception groulxiste de la nation est essentiellement culturelle, organique, providentialiste et messianique; qu'elle pense la nation comme préexistante à l'État; que, pour l'essentiel, elle n'a guère varié au fil des ans; et qu'elle implique une solidarité, en droit, indépendante du régime politique et, en fait, aussi active que possible. Les minorités canadiennesfrançaises font partie intégrante de la nation au même titre que les Canadiens français restés au foyer québécois et peu importe la province canadienne ou l'État américain où elles se sont établies. De cette définition de la nation par Groulx découlent ses idées concernant le renforcement du foyer national québécois, les responsabilités de ce dernier à l'égard 
des différentes composantes de la nation et l'attitude qu'il convient d'adopter à propos de l'autonomie provinciale et de l'indépendance. Je ne crois pas qu'il soit possible de contester globalement l'interprétation de l'auteur.

Des questions se présentent à l'esprit. L'auteur soutient que l'idéologie de Groulx «était issue, d'une manière générale, du romantisme politique du dix-neuvième siècle français» (p. ii). L'identité nationale telle que la pense Groulx lui apparait comme une " construction romantique et organique " (p. 343). Mais la filiation avec les auteurs français n'est pas établie de façon circonstanciée et détaillée. Le lien avec la philosophie politique allemande paraît encore plus ténu. L'exploration doit être poursuivie. On s'en convainc d'autant plus que l'on trouve sous la plume de Michelet, en 1831, des phrases comme celles-ci à propos de la nation française : «[...] une et identique depuis plusieurs siècles, elle doit être considérée comme une personne qui vit et se meut. Le signe et la garantie de l'organisme vivant, la puissance de l'assimilation, se trouve ici au plus haut degré $[. . .]^{10}$ »

Que Groulx recourait au droit positif et au droit naturel pour soutenir le combat de la survivance est indubitable. Mais on peut différer d'avis avec l'auteur, qui rattache la conception groulxienne du droit naturel à la philosophie romantique. Le droit naturel dont se réclame Groulx est plutôt celui de l'anthropologie catholique, qui s'enracine dans le thomisme, les encycliques papales et les œuvres des catholiques sociaux, y compris les auteurs de traités de droit public. Le droit naturel des romantiques est, en bonne partie pour les Allemands et essentiellement pour les Français, le droit naturel moderne, celui des Lumières, qui elles-mêmes ont puisé dans la pensée du XVII ${ }^{\mathrm{e}}$ siècle, celle de Locke, de Hobbes et de la Glorieuse Révolution de 1688-1689. Le matérialisme mécaniste et le pragmatisme libéral ne sont pas considérés par Groulx comme 
des références, même retravaillés par le mouvement nationalitaire de la Révolution française et du romantisme politique. Le droit naturel de Groulx est cette partie de la législation divine à laquelle donne accès l'exercice de la droite raison, sans le secours de la Révélation. Le droit naturel reste subordonné au droit révélé, au Décalogue et à son interprétation par la Tradition de l'Église. Son principe n'est pas l'autonomie morale et son système n'est pas l'individualisme. C'est plutôt le personnalisme chrétien d'avant Mounier : l'État est au service de la personne, mais l'individu ne saurait se concevoir valablement en faisant abstraction de sa condition d'être situé, inséparable de sa famille, de sa profession, de sa région, de sa langue, de sa culture et de sa nation. La convergence avec des traits du romantisme politique est évidente, mais ne nous autorise pas à les confondre. Soit dit en passant, Groulx n'accepte pas le principe révolutionnaire des nationalités. Plutôt que dans le romantisme politique plus ou moins allemand, ne faudrait-il pas chercher l'inspiration de Groulx dans le traditionalisme contre-révolutionnaire français corrigé par certains aspects du libéralisme catholique, français aussi, ainsi que du parlementarisme britannique, le tout retravaillé dans le contexte canadien et nord-américain, - donc dans un traditionalisme devenu indigène et maintenu dans le respect de l'anthropologie catholique et de la Doctrine sociale de l'Église ? Cela laisse néanmoins entier le problème des rapports entre le romantisme politique et la Contre-Révolution.

L'auteur a raison d'insister sur le fait que la conception de la nation chez Groulx invitait ce dernier à privilégier le fédéralisme. Il fait bien aussi de reconnaître que cette conception s'accompagne d'une grande liberté à l'égard des formes politiques, à travers lesquelles la solidarité peut toujours être sauvegardée conformément au volontarisme groulxien. $\mathrm{Si}$ toutefois l'on distingue le niveau du mythe, horizon vers 
lequel l'esprit et la volonté aiment à se tourner, et celui de la pratique, force est de reconnaitre que l'idée d'indépendance chez Groulx joue un rôle plus grand que celui que concède l'auteur. Cela dit, je n'ai vu exposer nulle part ailleurs de façon aussi forte et aussi documentée l'antinomie entre le nationalisme canadien-français de Groulx et le néonationalisme québécois de ses disciples, en particulier ceux de l'École historique de Montréal. Ces derniers, modernisant la discipline historique, en bannissent le providentialisme et justifient par l'objectivité scientifique leur abandon des minorités françaises. Leur conception de la nation se transforme et, au terme de la transition, elle aura répudié le romantisme organique pour souscrire au rationalisme territorial.

Le groulxisme que, de main de maitre, nous décrit Michel Bock n'est pas un tissu d'incohérences, de palinodies et d'absurdités, mais une pensée vivante et par conséquent animée, comme tout vivant, soit par des tensions internes, nées de ses polarités, soit par des tensions externes, chocs en retour de l'environnement et de son évolution. La rationalité de cette pensée se laisse saisir dès lors que l'analyste du discours se tient loin de la déchiqueteuse et ne confond pas fragments et confettis. Les travaux de Frédéric Boily le corroborent.

«Le trait dominant et essentiel du groulxisme, c'est la croyance fondamentale en un organisme national qui vit et se développe à travers l'histoire ${ }^{11} »$ (p. 181). Voilà la thèse de Frédéric Boily, qui, pour cette raison, voit dans l'organicisme ou le nationalisme organiciste la clé de la pensée de Groulx (pp. 19, 21). Comme il considère que Groulx réussit à préserver une réelle intelligibilité au sein de son ambivalence, il lui semble raisonnable et utile de chercher «le fil conducteur de son œuvre pour en retrouver la cohérence interne » (p. 19). Le nœud, c'est la conception groulxienne de la nation, qui fournit à l'ensemble à la fois sa logique - incontestable — et son 
ambivalence. Cette dernière s'enracine radicalement dans la manière de penser la nation chez Groulx, qui « combin[e] logique raciale et culturalisme nationaliste » (p. 21), avec toutefois nette prédominance du second sur la première (p. 210).

L'organicisme conçoit la nation et la société comme des totalités organiques, des êtres collectifs vivant chacun comme un individu. L'auteur passe en revue ce qui lui paraît les grands thèmes et les articulations principales du groulxisme pour les éclairer à la lumière de l'organicisme : définition et historicité de la nation, rapport du national et du religieux, construction du récit national ou de la biographie de l'Être national, relation de la nation et de l'État, intellection du politique, rôle du chef, de l'élite, de l'intelligentsia, théorie de l'éducation nationale et de la place de l'histoire nationale. Un ultime chapitre débusque l'héritage groulxiste chez divers penseurs, de Maurice Séguin à Charles Taylor et Gérard Bouchard. La conclusion constate que la systématique groulxienne livre son secret à qui l'interroge sous l'angle organiciste et que, en 2003 autant qu'auparavant, l'étude de Groulx importe à la compréhension du Québec contemporain.

L'exploration de la structure et l'inventaire des traits organicistes chez Groulx se réalisent à l'aide d'une comparaison avec des penseurs européens, en particulier Herder et Fichte, Herder surtout. Groulx ne lisait ni l'allemand ni les auteurs allemands. Boily se justifie en rappelant que, « en Europe, la pensée herdérienne a servi de modèle à tous ceux qui par la suite se sont engagés dans un processus de construction nationale » (p. 23). J'accepte cette astuce analytique en raison de sa relative fécondité et parce que le jeu des filiations n'est pas le propos central de l'auteur. Mais j'ai deux réserves. Si l'auteur avait placé son enquête à l'enseigne du traditionalisme organiciste, il aurait pu s'en tenir aux intellectuels que Groulx a effectivement lus et même étudiés. Herder 
n'a inauguré ni l'organicisme, ni le traditionalisme, les deux plongeant leurs racines dans le Moyen Âge et même l'Antiquité. Sans remonter aussi haut, Burke, Maistre, Bonald et Lamennais ont exercé une réelle influence au Canada français, directement ou par commentateurs interposés. Je concède que, pour le chercheur, la pensée de Herder est séduisante parce qu'elle atteint à un degré d'achèvement commode. Je ne récuse donc pas le choix de l'auteur. Ici intervient ma seconde réserve. Dans cette étude, Groulx se présente au lecteur comme plus ou moins herdérien, fichtéen ou barrésien, etc. À tout coup, la confrontation est incomplète. L'auteur recense les ressemblances entre Groulx et ses "inspirateurs » sans s'embarrasser de l'établissement des différences. Entre un catholique ultramontain comme Groulx et des penseurs protestants, déistes ou agnostiques en dialogue avec les $\mathrm{Lu}$ mières, il y a suffisamment de distance pour qu'on demande à $\mathrm{y}$ regarder de plus près.

Les deux chapitres les moins convaincants sont le deuxième et le sixième, sur "l'instrumentalisation nationaliste du catholicisme " et sur "la conception de l'éducation chez Groulx ». Dans le premier, Groulx marche sur la tête. En réalité, la religion détient une réelle primauté dans l'univers groulxien. Une position moins tranchée, mettant en scène la dialectique du religieux et du national, aurait été plus fidèle à la pensée de Groulx. Il arrive à l'auteur d'être plus nuancé (la réflexion de ce chapitre se poursuit ailleurs : pp. 81, 91, $110,120,122,130,133,135,141,148,157,167)$. Je déplore une sous-estimation des fondements religieux du monde des idées tel que Groulx se le représente. Vers la fin du livre, l'auteur souligne le fait que la nation groulxienne est « un véritable "être moral" » (p. 181). Cette notion aurait appelé une discussion car elle nous aide à mieux saisir les contours, les limites de l'organisme national. De même, le thème de la modernité 
est trop discret (par exemple, pp. 130, 157, 201 et 206). Groulx est philosophiquement antimoderne parce qu'il condamne le déplacement du théocentrisme par l'anthropocentrisme. Je trouve bien aventurées les quelques lignes par lesquels l'auteur voudrait cerner le statut de la vérité dans le groulxisme (p. 133).

On trouve dans le chapitre VI des défauts qui ne sont pas toujours absents des autres. L'auteur n'analyse vraiment que les textes de Groulx relatifs à l'éducation nationale, mais il se permet de se prononcer sur la théorie générale d'éducation que l'on trouve chez lui. En substance, selon l'auteur, Groulx ne voulait pas apprendre à la jeunesse à penser par elle-même parce que sa priorité, c'était le dressage de cette dernière à la tradition nationale. Voilà qui me paraît bien catégorique. Ce résultat est obtenu au moyens de glissements et de dichotomies. Glissement, par exemple, de l'éducation nationale à l'éducation en général (p. 166) comme si l'éducation nationale était le tout de l'éducation pour Groulx. Il faut rappeler que la nation est, pour ce dernier, une nature seconde, qui n'oblitère pas la nature première et fondamentale de l'homme. On observe de tels glissements ailleurs, par exemple du prêtre singulier que Groulx était, au prêtre-éducateur et au prêtre tout court (pp. 135-136).

L'analyse est souvent dichotomique, à la manière de Cité libre et de Max Nemni. Si $A$, non $B$; si $B$, non $A$. Cette logique binaire régente trop la discussion. L'auteur enferme le réel dans des oppositions artificielles, au lieu de poser des polarités auxquelles aboutit un continuum. En éducation, ou vous êtes pour Kant, ou vous êtes pour Fichte. Je ne suis ni philosophe, ni germaniste, mais j'avoue ne reconnaitre tout à fait dans ce livre ni Fichte, ni Kant, ni même Herder. Mais là n'est pas la question. Je regrette que l'auteur, par sa méthode même et sans le vouloir, appauvrisse la pensée de Groulx. 
Sa discussion des rapports entre le groulxisme et le séguinisme n'est pas non plus satisfaisante. Il a tendance à réduire le conflit à une question de vocabulaire. Le radicalisme de Séguin lui échappe : si ce dernier garde de la nation une conception ethnique, il lui fait subir une métamorphose qui constitue une rupture fondamentale avec la nation groulxienne. Le nationalisme séguiniste s'obtient par une laïcisation de la nation providentielle et par la substitution du structuralisme au volontarisme. Ce n'est pas seulement la Conquête qui est réinterprétée, mais l'ensemble des rapports entre la personne, agent libre, et la nation, dont le destin est gouverné par les structures de l'histoire, domaine de la nécessité. L'indépendance nécessaire mais impossible appelle un dépassement dialectique, qui, à ce jour, n'a été réussi ni dans la pensée, ni dans l'action. Groulx ne croyait pas que l'indépendance était impossible parce qu'il n'en avait pas une définition aussi intransigeante que Séguin, même si son rêve, au fond, était plus grand et plus exaltant.

Remarquant que par deux fois (pp. 135 et 143), l'auteur renvoie erronément au chapitre III au lieu du chapitre II, je me suis reporté à la thèse dont la présente étude est tirée. Le plan en a été réaménagé. J'ai pu lire le parallèle fragile entre Groulx et Heidegger que l'auteur a supprimé dans son livre, "par souci de présentation et de clarté ", précise-t-il (p. 129). C'est une sage décision. J'observe aussi que l'auteur manifeste plus de prudence et de retenue; que sa démonstration est mieux maitrisée.

Malgré les réserves que j’ai exprimées - et il y en aurait d'autres —, je persiste à croire que cette étude projette des clartés assez généreuses sur Groulx et le groulxisme pour nous engager à dialoguer avec son auteur. Ce n'est pas assez dire : il faut saluer cette contribution importante à la connaissance de Groulx, l'une des plus solides depuis le livre de Jean-Pierre 
Gaboury, qui est de 1970. Si le but de l'auteur était de montrer que l'organicisme rend compte davantage du groulxisme et de sa structure profonde, avec plus de profit pour l'intelligence et plus d'attrait pour l'esprit, que le fascisme, le racisme, l'ethnicisme et l'eugénisme, il a gagné son pari et prouvé sa thèse. Il apporte et cimente des pierres bien taillées à l'édifice commun et il peut compter que ce dernier ne s'achèvera pas sans lui. C'est un livre intelligent et serein, représentatif de la nouvelle génération de politologues, de sociologues et d'historiens qui scrutent le passé canadien-français en toute liberté quoique sans complaisance, dégagés qu'ils sont des impatiences et des amertumes des plus âgés. Le plaisir de lire y gagne ; la science, encore plus.

Le dernier chapitre du livre de Boily, qui s'interroge sur le prolongement du groulxisme jusqu'à aujourd'hui sous la forme d'influences non avouées la plupart du temps, en a fait sourciller quelques-uns. Cette ouverture sur un questionnement à entreprendre ne visait pas à donner des réponses définitives. Qu'y a-t-il de mal à soulever des questions, dès lors qu'elles sont pertinentes et nourrissent la réflexion ? Les quelques pages consacrées à Gérard Bouchard suggèrent une homologie des conceptions bouchardiennes et groulxiennes des rapports entre l'historien, la nation et le passé. Délaissant les études sur les populations et l'histoire sociale, le sociologue se consacre désormais à l'analyse des représentations. Il espère pouvoir ainsi mieux influer sur les débats et le devenir de la nation, en fait sur la naissance d'une nation nouvelle, ni ethnique, ni civique, mais sociopolitique et inclusive, où un certain culturalisme canadien-français se maintiendrait néanmoins obscurément. Il a même publié son Appel de la race, à moins que ce ne soit son $A u$ cap Blomidon, sous le titre de Mistouk $k^{12}$. Autant d'indices de l'aspiration à succéder à Fernand Dumont et surtout à Groulx. Bouchard louange Groulx quand 
ce dernier se rapproche des propositions de La nation québécoise au futur et au passé; il le réprimande quand il s'en éloigne $^{13}$. En battant la grosse caisse devant les contradictions de Groulx, réelles, appréhendées ou imaginaires, il fomente une rupture par le discrédit. Tant que des lambeaux de groulxisme resteront accrochés aux arêtes de la conscience nationale, le penseur n'aura pas ses coudées franches et il ne pourra pas revêtir la toge, à défaut de la soutane, de l'historien national.

Il est permis d'avoir des ambitions. Mon interrogation porte sur le type de pensée que distille Bouchard avec le brio que tous reconnaissent. Dans Les deux chanoines, il distingue la pensée radicale, la pensée organique et la pensée équivoque ${ }^{14}$. Éliminons la pensée radicale. Il lui faudrait être moderne en toute logique, déserter les terres de la nation comme communauté mémorielle - même retrempée à l'antique dans une rafraîchissante généalogie amérindienne - et, chantre de l'individu, batailler ferme pour la République universelle, en commençant peut-être par une Confédération nord-américaine. Ce n'est pas son programme. Même s'il n'aime pas le mot, la chose lui est chère, et le sociologue romancier reste viscéralement nationaliste, bien que sa nation soit problématique puisqu'elle n'est pas encore née. Écrire l'histoire d'une nation même à naître, c'est encore s'adonner à l'histoire nationale. La Révolution tranquille a eu beau régénérer tout cela, la palingénésie péquiste et postmoderne tout restaurer et tout perfectionner, le nationalisme à la Bouchard mime le traditionalisme, l'obscurité en prime. Il prétend à la fois rompre et continuer. Les ambivalences, les ambiguïtés, les équilibres instables entre contraires, les raccourcis enfin des essais de Bouchard n'annoncent pas encore les dépassements-synthèses par fécondité dialectique que désigne la pensée organique, formule vieillotte qui fleure le collège classique. Alors, oui, hélas! il reste la pensée équivoque. Le sociologue, qui s'est 
mesuré au dur labeur d'accoucheur de peuple, sait de quoi il retourne. C'est le renard de la fable, qui, ayant la queue coupée, souhaite que chacun l'imite. Or il ne sera peut-être pas si facile d'écourter, selon le mot de La Fontaine, Groulx le renard.

La thèse de l'auteur est aussi simple que grosse : Lionel Groulx est l'homme-contradiction. Son univers intellectuel est étrange, «foncièrement incohérent » (p. 10). Quand donc ? Question superflue car, comme il n'a pas évolué, la chronologie importe peu. S'il échappe à la contradiction, c'est pour s'enliser dans l'ambiguiité ou se complaire dans l'ambivalence. Sa longue vie y a passé, le pauvre. Penseur nationaliste, il n'a même pas pu penser la nation (p. 13). Boily n'a rien compris. Pour démonstration, Bouchard nous sert 250 pages de sa prose baleinée de rectitude politique, alourdie de pédagogie civique et encombrée de présentisme. Tout y passe : Groulx le traditionaliste a produit un néonationalisme et un révisionnisme historique ; providentialiste délirant, il était moderne et conservateur, libéral et réactionnaire, non essentiellement raciste, mais assez souvent raciste tout de même, en tout cas ethniciste et eugéniste, antisémite plutôt non raciste et pourtant mystérieusement responsable du sort des Juifs d'Europe, dont il ne s'est même pas excusé, l'infâme - mais bon prince, l'auteur consent à laisser son nom figurer sur une des stations du métro montréalais -, écartelé entre la France et l'Amérique, impuissant mais pondeur de mythes à répétition, etc. : voilà son cas réglé, en attendant de montrer que, loin d'être solitaire, il appartenait à une tribu fort populeuse, qu'un mal, non, un bien, qui s'appelle Révolution tranquille, a heureusement décimée. Mais, cela, c'est pour le prochain livre.

Revenons à Groulx : « La contradiction, encore une fois, imprègne et structure toute sa pensée » (p. 13). Ceci encore : "Groulx n'était capable de penser efficacement ni le statu quo 
ni le changement, ni la tradition ni la modernité, ni la continuité (francité) ni la rupture (américanité)» (p. 237). (Soit dit en passant, cette phrase d'apparence limpide, caractéristique de la prose de Bouchard, ne signifie rien à cause d'un mot: efficacement. Qu'est-ce que cela veut dire ? Comment la sociologie peut-elle objectiver des appréciations aussi globales? Quels en sont les critères et les normes? L'an de grâce 2003 n'est pas un promontoire. Le point d'observation se déplaçant, le paysage s'anime et change en même temps que ses coordonnées. Où le point fixe, cher collègue ? Dans l'idéologie? Une idéologie sortit de grand matin pour en juger une autre...) L'auteur y insiste : "La part de contradiction dans la pensée de Groulx m'apparaît non pas comme marginale mais centrale, non pas accessoire mais structurelle. [...] En d'autres mots, Groulx m'apparaît comme le porte-parole d'une société, ou plus exactement d'un fragment de société, qui n'arrivait pas à se penser clairement ni à se poser efficacement dans l'histoire à partir de ses prémisses culturelles » (p. 21). On aura compris que Groulx est observé comme spécimen d'une société et non en lui-même : "ce sont moins les dispositions individuelles que les coordonnées collectives qui retiennent mon attention. Moins le penseur que la pensée comme fait social, dans ce qu'elle peut nous apprendre à la fois sur le fonctionnement de l'imaginaire, sur la structure d'une société et sur le cours de son histoire » (p. 256). Entendons-nous bien : l'hypothèse que veut vérifier l'auteur, la thèse qu'il se propose d'établir, le cadre où il entend inscrire sa démarche sont parfaitement légitimes. J'étais prêt à l'entendre et à soupeser avec lui, mieux me laissant guider par lui, ses arguments et ses documents, même si une crainte m'effleurait : le risque d'un retour aux simplifications de la sociologie à la Marcel Rioux, aux exécutions sommaires de La Grève de l'Amiante de Pierre Elliott Trudeau, aux a priori, évidences et certitudes 
des révolutionnaires-tranquilles, dont on commence à se désengluer. Ce serait plus raffiné, plus savant - la connaissance n'a pas progressé pour rien -, mais cela serait-il fondamentalement, structurellement différent?

Esther Delisle ne nous déçoit jamais parce qu'on n'en espère rien. Gérard Bouchard, c'est différent. La moindre de ses publications nous arrive précédée de la réputation, de la renommée, du prestige de ce savant prolifique. Un certain public recueille ses paroles comme des oracles. Qu'on juge de ma déception, de mon désarroi au fur et à mesure que je tournais les feuillets de son dernier essai. La problématique m'était apparue excellente au premier coup d'œil, ambitieuse au point de vue heuristique, bref digne de l'auteur. Mais en regard, la preuve et l'argumentation se révèlent d'une pauvreté inimaginable, je le dis en toute candeur. La démonstration est un échec.

Inutile de résumer davantage l'ouvrage. Il vaut mieux aller au plus court et donner une idée sommaire des raisons de cet échec. Le lecteur voudra bien m'accorder que j'ai du mérite à le faire. Car notre auteur est un original : les faits, phénomènes et fragments dans son texte ne sont pas ordonnés par périodes, mais les renvois des notes, eux, sont classés en ordre chronologique croissant plutôt que selon l'ordre d'apparition des citations. Dans ces conditions, retrouver une référence exige de la patience.

Personne ne prétendra que dans la pensée de Groulx comme dans toute pensée d'ailleurs - des ambivalences, des ambiguités, des incohérences, des tensions entre contraires, des apories ne puissent se rencontrer. Elles s'y rencontrent en effet. Mais l'auteur ne parvient pas à établir qu'elles sont constitutives du plus clair de la pensée de Groulx. Cette faillite de la preuve vient de lacunes de méthode pour moi incompréhensibles chez un chercheur aussi chevronné, d'une incapa- 
cité à penser logiquement, d'une faiblesse analytique étonnante. L'auteur veut prouver que les contradictions sont la pensée de Groulx. On a le droit d'exiger qu'il soit capable d'identifier la contradiction, donc qu'il en connaisse la définition et qu'il sache raisonner. La stupéfaction attend le lecteur de Bouchard.

La contradiction est l'opposition la plus radicale entre deux énoncés. Le principe de contradiction dénonce une opposition radicale sous le même rapport. Les implications pour la méthode sont considérables, inéluctables. L'histoire intellectuelle a depuis longtemps appris à se méfier de la polysémie, non pas celle du dictionnaire, mais celle qui dépend du locuteur situé dans le temps, dans l'espace et dans l'environnement épistémologique et idéologique. L'intellectuel, en particulier, travaille et retravaille ses concepts, ses termes et ses notions à la lumière des sollicitations du moment et des exigences de sa réflexion, elle aussi traversées par le temps. La polysémie est fonction des contextes : le contexte structurel particularise les termes (les libertés libérale, libertaire, fasciste, communiste, catholique, athée répondent toutes au beau mot de liberté ; la démocratie parlementaire libérale garde son nom qu'elle repose sur le suffrage censitaire, masculin ou universel); le contexte conjoncturel est le moment et le lieu où se croisent et se heurtent les dimensions de l'existence, les requêtes de l'actualité et les désirs de la personne; le contexte textuel désigne l'ensemble des relations qu'entretiennent entre eux les énoncés au sein d'un même texte et d'un texte à l'autre, dans la simultanéité et dans la mémoire, en un fantastique jeu de miroirs et de mises en abyme. Par conséquent, toujours se demander quelle interrogation, intérieure ou extérieure, quelle référence, quelle provocation, quel tourment, quel discours le locuteur avait en tête et le faisait réagir. X se dit démocrate en 1990 et non démocrate en 2000 : 
contradiction ? Énoncés contraires, très certainement. Contradictoires, oui, si dans les deux cas démocratie se prend sous le même rapport et dans le même sens, car $\mathrm{X}$ peut récuser la démocratie comme état social égalitaire parce qu'il est élitiste et admettre la démocratie en tant que mode de désignation des gouvernants. Les trois types de contextes exigent la chronologie et la périodisation; le jugement sur les contradictoires et les contraires doit faire intervenir les contextes. Or l'auteur se dispense des unes et des autres au motif que même « en matière de principes, d'idéaux, de prémisses, Groulx affirmait simultanément le blanc et le noir» (p. 197) et au motif encore que l'auteur laisse à d'autres la micro-histoire intellectuelle. Si l'on reçoit ce dernier motif, l'on est en droit d'intimer à l'auteur de s'en tenir aux contradictions patentes, flagrantes, indiscutables puisqu'il a renoncé au droit de se prononcer en connaissance de cause sur les autres. La prudence s'impose d'autant plus que la pratique sociologique de l'auteur est surdéterminée par la dynamique d'intégration au détriment de la dynamique d'opposition, qui pourtant l'équilibrerait. Et, chez lui, la dynamique d'intégration se fait volontiers normative.

Une maîtrise déficiente du principe de contradiction affaiblit l'argumentation de l'auteur, qui est aussi menacée par la négligence du principe de raison suffisante. Pour identifier une réalité, il faut en réunir les conditions de nature nécessaires et suffisantes. Dans le maniement des définitions et l'analyse de ses fragments, l'auteur erre de temps à autre. De quelques indices ou de quelques traits définitoires, il conclut à la présence ou non de la chose. Inversement, parce que tel renard n'a pas de queue, est-il moins renard pour autant ? L'argumentation de l'auteur repose parfois sur des sophismes, qu'il faudrait appeler paralogismes car il est de bonne foi. Outre le sophisme de l'opposition, qui confond contradictoire et con- 
traire, comme on l'a vu, on trouve le sophisme du dénombrement imparfait (ou énumération insuffisante), qui ne considère pas assez les différentes manières d'être ou de se produire d'une chose ; le sophisme de l'accident, qui substitue l'accidentel à l'essentiel, la partie au tout; le sophisme d'équivoque verbale, qui prête deux sens au même terme dans le cadre d'un même raisonnement, ou bien glisse du sens divisé au sens composé, ou encore du sens propre au sens figuré. L'auteur lit parfois ses citations à travers l'un ou l'autre de ces paralogismes. Ces erreurs de jugement échappent à la lecture cursive; elles crèvent les yeux lorsque les citations sont vérifiées. Cette vérification est d'autant plus nécessaire que la citation peut être réduite à sa plus simple expression : un syntagme, un mot.

Si la crédibilité de l'auteur s'effrite au fil de la lecture, même chez le lecteur pressé, c'est qu'il en fait trop et manque de jugement. Il verse le contenu de ses hottes. Devant les tas où se mêle tout un bric-à-brac de bouts de ficelle et de clous rouillés, il triomphe à bon marché. Mais le passant s'éloigne. Or ce dernier a tort : s'il remuait du bout de son bâton tout ce disparate, il verrait briller du bon or, pur et massif. Seuls la problématique et cet or méritent considération et discussion. Je souhaite que l'auteur se discipline et se reprenne : passé au crible, enrichi d'un apparat critique pratique et correct, délesté de la moitié de ses pages, l'ouvrage pourra soutenir des échanges fructueux entre historiens, sociologues et politologues. D'ici là, aussi bien se taire.

C'est à Frédéric Boily que le collectif Les visages de la foi a confié le chapitre consacré à Groul ${ }^{15}$. Malheureusement, le lecteur doit se contenter d'un sous-produit de sa thèse de doctorat. L'auteur se borne à présenter l'interface politiquereligion, pour parler comme un fonctionnaire québécois. Même le volume Rencontres avec Dieu est interrogé de cette manière. Il 
aurait dû servir à nous introduire au plus intime de la croyance de Groulx, savante en même temps que populaire, bérullienne aussi dans son insistance sur le Verbe incarné. Inutile d'espérer une analyse de quelques sermons inédits. Les auteurs de l'introduction, globalement excellente, singularisent Lionel Groulx et Marie-de-la-Recouvrance : « Ce sont des noms dont le témoignage n'est pas bien net, c'est le moins que l'on puisse dire, et pour un peu on le récuserait tout entier s'ils ne faisaient pas d'ores et déjà partie de l'histoire » (p. 31). Et si, pour être "bien net», le témoignage exigeait le traitement hagiographique ? Bien net, le témoignage du gouverneur général Vanier? du frère Clément Lockquell ? du père GeorgesHenri Lévesque? Exagérée, la démarcation de l'avant et de l'après 1945, un peu oublieuse des constantes en tension au sein du christianisme? Et cette tendance à qualifier de personnaliste l'éthique ou la contemplation d'une certaine époque : abus de vocabulaire ? Pourquoi n'y a-t-il pas de scientifique parmi les témoins de la foi ici rassemblés? MarieVictorin est sans doute simul justus et peccator, et certains jours davantage peccator que justus, mais ses textes sur la foi valent le détour. Dans la catégorie des prophètes, pourquoi avoir exclu le seul qui soit ainsi désigné par Jean Hamelin, le père Onésime Lacouture, prophète persécuté par un clergé embourgeoisé peu soucieux de se faire rappeler certaine parole un peu forte de saint Paul ${ }^{16}$ ?

Boily connaît-il assez le catholicisme, maîtrise-t-il assez le contenu intellectuel de la foi chrétienne pour se prononcer sur le caractère pessimiste ou non de la doctrine du péché originel chez Groulx ? Je lui suggère de s'absorber quelques heures dans le Catéchisme de l'Église catholique ${ }^{17}$. Pourquoi, dans un texte essentiellement politique, Groulx remonte-t-il jusqu'au péché originel ${ }^{18}$ ? C'est qu'il répond à l'abbé Arthur Maheux qui entend prouver que le bonne-ententisme est évan- 
gélique et le mouvement qui s'en inspire, providentiel ${ }^{19}$. Maheux a choisi son terrain. Groulx l'y affronte. Et, dans l'exégèse de Groulx, tendre la joue gauche n'a jamais voulu dire se résigner à l'oppression et au mépris. Le temps de l'abbé Maheux, c'est celui dont le grand écrivain anglo-juif de Montréal Mordecai Richler avait la nostalgie, les années 1945-1960, le temps béni d'avant la Révolution tranquille et d'avant la Loi 101 : "Anglophone nostalgia for an earlier Montreal is not necessarily based on a longing for economic dominance but rather for a time when English, as well as French, thrived there and the two cultures enriched rather than excoriated one another ${ }^{20}$. » Quand la langue française était bafouée, l'harmonie était si facile et la cohabitation culturelle si enrichissante.

Boily a lu peu de chose sur la religion de Groulx. Il n'a pas toujours bien lu. Courant après l'esprit, il tient à nous révéler que Groulx condamnait une alimentation trop riche en lait maternel (p. 175). Il ne donne pas de référence précise. C'est dans Chemins de l'avenir, à la page $11^{21}$. Se fondant sur l'opinion d'un médecin français, Groulx dit exactement le contraire. De même, dans un article qui est loin d'être sans mérite, je le reconnais volontiers, Sylvie Beaudreau me fait dire qu'en tant qu'historien Groulx a été surestimé (p. 32) ${ }^{22}$. C'est plutôt l'opinion de Robert Rumilly, que tout mon article réfute. Insignifiance, sans doute, mais qui ébranle la confiance à l'égard de l'historien ou du politologue qui s'adonnent à l'analyse textuelle.

Sylvie Beaudreau montre que le nationalisme de Groulx est un spiritualisme, ce qui en fait non pas un homme d'action frustré, mais « un homme d'action spirituelle intensément satisfait» (p. 61). Comprendre : satisfait de s'en tenir à l'action spirituelle et intellectuelle, mais nullement satisfait des résultats obtenus. Ne se méfiant pas suffisamment des cli- 
chés (homme de la Renaissance, homme du XVII ${ }^{\mathrm{e}}$ siècle), elle réduit la pensée nationale de Groulx à un protonationalisme et son État français à un état d'esprit. Ce n'est pas qu'un état d'esprit. C'est un État déjà à demi-structuré, sur un territoire parfaitement objectif, et doté d'institutions parlementaires tout aussi concrètes depuis la fin du XVIII ${ }^{e}$ siècle. Ce qui, à cet égard, était possible au Québec n'était tout simplement pas envisageable dans la diaspora.

Pour admettre toutes les conclusions de Sylvie Beaudreau, il faudrait partager sa naïveté. Elle semble croire qu'une seule Révolution tranquille était possible et que la réforme de l'éducation ne pouvait être différente. Contrairement à Groulx qui était obnubilé par sa conception de l'homme (une âme et un corps substantiellement unis), les commissaires du rapport Parent auraient été en contact immédiat avec les besoins objectifs de la société, bénéficiant du privilège exceptionnel de l'affranchissement de toute médiation idéologique. Ainsi ce ne sont pas deux idéologies qui s'opposent, mais l'idéologie et le réel. L'ambivalence de Groulx envers la Révolution tranquille s'explique, selon elle, non pas par des contradictions internes à son discours, mais par des contradictions externes «entre un proto-nationalisme typique du $\mathrm{XIX}^{\mathrm{e}}$ siècle et le néo-nationalisme de la Révolution tranquille» ou encore « entre une conception de l'identité catholique de la nation enrichie par une mystique tant personnelle que nationale, et les aspects technocratiques, bureaucratiques, sociologiques et laïcisants du programme de modernisation proposé par le gouvernement Lesage» (pp. 59-60). En somme, elle soutient que le nationalisme de Groulx était apolitique, mais néanmoins partisan de l'édification de l'État. Elle oublie que, à partir des années 1790, le nationalisme canadien-français s'est développé dans le cadre d'un État colonial caractérisé par ses institutions parlementaires, bien qu'elles étaient 
encore inchoatives. Même si elle s'en défend, elle raisonne comme si la nation culturelle et la nation politique étaient antinomiques et comme si tout nationalisme qui ne s'oriente pas vers la constitution immédiate d'un État-nation indépendant cessait d'être moderne et, par là même, rétrogradait au stade protonationaliste. Les fédérations seraient-elles des réalités archaïques ? C'est nous ramener aux interprétations des années 1950-1980, dans lesquelles les sociologues et les historiens opposaient sans nuance une caricature du nationalisme traditionnel ou culturel au nationalisme politique, feignant de croire que l'État québécois n'était qu'un fantôme et que l'Église régnait sans partage. L'auteur a raison d'opposer l'identité catholique groulxienne et la sécularisation - Groulx est antimoderne en ce qu'il récuse la modernité laïque, qu'il faudrait définir d'ailleurs —, mais on voit mal comment cette identité empêchait l'État groulxien de se moderniser et de se bureaucratiser. À moins, bien entendu, qu'il soit défendu d'envisager toute modernisation autre que celle que le Québec a connue. Groulx ne s'intéressait pas seulement à l'État du point de vue de l'affranchissement économique ; la politique économique ne lui interdisait pas l'accès au politique ; le fédéralisme lui paraissait avoir des avantages pour les petits peuples, mais il se refusait à le mythifier. La singularité du parcours québécois ne s'accommode pas aisément des catégories d'Eric Hobsbawm. Il faut se garder de penser que l'on raisonne sur des réalités historiques quand on considère plutôt les éléments d'une typologie théorique, surtout quand elle a été pensée en fonction d'autres contextes.

C'est dans les premiers jours de décembre 2003, donc bien après la lecture des Deux chanoines, que je me suis plongé dans la thèse inédite de Norman F. Cornett, de la Faculté des études religieuses de l'université $\mathrm{McGill}^{23}$. Je l'ai abordée avec respect, sachant qu'elle repose sur plus de quinze ans d'ex- 
ploration en profondeur et de réflexion soutenue. Mais l'honnêteté m'oblige à confesser que je n'étais pas rassuré. Groulx a un curieux effet sur certains intellectuels, qui n'est pas sans rappeler la maxime des anciens : "Quos vult perdere Deus dementat prius», Dieu prive d'abord de raison ceux qu'il a résolu de perdre. Avant de lire l'ouvrage, pour m'enhardir, je l'ai feuilleté. Je suis tombé sur ces mots : «Groulx's worldview was essentially Augustinian, with a Thomist twist» (p. 187). Cela a suffi à me rassurer. Cet énoncé suppose une connaissance intime de Groulx. Il explique l'extraordinaire ouverture de ce dernier au néoscotisme d'André Dagenais, attitude de souplesse qui défierait l'entendement de la part d'un intégriste thomiste ${ }^{24}$. Il y a dix ans, j'écrivais que, parmi les intellectuels traditionalistes, le philosophe André Dagenais était «le seul à avoir explicité les bases métaphysiques de sa doctrine et par là-même de la droite canadienne-française en général ${ }^{25}$ \%. Le professeur Cornett montre magnifiquement que Lionel Groulx, continuant $\mathrm{M}^{\mathrm{grs}}$ Laflèche et Pâquet, en a explicité les bases théologiques. "L'homme, scande Jean de Viguerie, est un être pensant et croyant ${ }^{26}$.»

J'ai donc lu et j’ai été ébloui. Comme je retrouvais fréquemment au fil de la lecture des interprétations semblables à celles de mes notes et brouillons préparatoires à la biographie que je me propose de consacrer à Groulx, je me suis gardé de toute complaisance et je me suis efforcé de maintenir en éveil mon sens critique. Peine perdue, si j’ose dire : ma satisfaction et mon plaisir sont allés croissant. Je n'aurais pu, je ne dis pas: faire mieux, mais simplement faire aussi bien. Si un cataclysme s'abattait sur les bibliothèques et que, dans ma fuite, je ne pusse emporter qu'une étude groulxienne, j'empoignerais celle du professeur Cornett, convaincu de sauver le Groulx essentiel. Il faut publier en anglais et en français The Role of Religion in Lionel Groulx's Nationalist Thought. Fides, l'éditeur de Groulx, doit saisir l'occasion. 
L'auteur nous fait partager sa conviction que la religion constitue le cœur, l'âme, la structure profonde et explicative de la pensée de Groulx. Plus précisément, il démontre, au moyen d'une preuve fortement documentée et solidement charpentée, que le prêtre-historien nationaliste a fondé sur le mystère de l'Incarnation, irruption de Dieu dans le temps humain et dans l'histoire des hommes, son interprétation du passé et du devenir de la nation canadienne-française, sa version du nationalisme et la raison d'être du combat de toute sa vie. Poussé par la formation religieuse reçue à l'école et au collège, porté par la pratique des Écritures, y compris l'Ancien Testament, confirmé par ses études philosophiques et théologiques essentiellement thomistes et maintenu dans cette voie par sa fréquentation persévérante, jusqu'à sa mort, des ouvrages de spiritualité et de science religieuse, Groulx fait du mystère de l'Incarnation la méditation de toute son existence et l'enracine dans la vie nationale en en déduisant le paradigme de l'incarnation.

Ce paradigme est la clé d'or pour pénétrer le groulxisme et pour y rendre intelligible la dialectique de l'universel et du particulier, du religieux et du temporel, du sacré et du profane. Déroulant les enchaînements de la thématique sotériologique - une sotériologie davantage communautaire qu'individuelle et sans dissociation du salut éternel et du salut temporel —, Groulx va de l'Homme-Dieu à l'Église, corps du Christ en même temps que nouveau peuple de Dieu, puis de l'Église à la nation canadienne-française, nouvel Israël en terre d'Amérique. L'unanimité confessionnelle du Canada français, unique en Amérique du Nord, est le signe de l'élection divine, d'une mission providentielle de témoin par excellence de l'Incarnation. Car si des catholiques de toutes origines dressent des clochers dans toutes les villes du continent, aucun autre peuple que les Canadiens français ne peut revendiquer 
le terrible privilège d'incarner de façon aussi plénière la foi, l'éthique et l'éthos du catholicisme. Au Québec, où ils sont majoritaires, ils n'ont qu'à le vouloir pour édifier une civilisation catholique exemplaire. D'où, selon la sagesse biblique, le devoir de "sainteté » des Canadiens français, donc de "séparation» des autres peuples, en somme de fidélité intégrale à leur Dieu, à leur mission et à leur être profond. C'est dans l'entre-deux-guerres que cette version du paradigme est affirmée avec le plus de démonstration, de force et d'autorité. Cette période coïncide, très logiquement, avec la haute époque de l'engagement nationaliste chez Groulx. Son attachement à sa conception de l'Action catholique comme mouvement indissociablement religieux et national se comprend mieux sous cet éclairage.

La montée de l'anticléricalisme et la sécularisation, discrète puis ouverte, du Canada français dans l'après-guerre troublent profondément Groulx, qui révise son paradigme de l'incarnation. La Révolution tranquille clôt l'ère de la chrétienté canadienne-française. Il le reconnait. Comme le peuple juif jadis, la nation canadienne-française a démérité et il convient de redéfinir sa vocation, qui apparaît du coup moins exceptionnelle. Il semble qu'elle soit réduite au rôle commun des autres groupes catholiques, ce qui néanmoins l'oblige toujours à témoigner et à répandre la foi dans le monde. Ces dernières décennies de la vie de Groulx sont précisément celles où l'inquiétude catholique devient première et le nationalisme, second, et où l'action proprement nationale s'estompe. Groulx ne répudie ni l'Incarnation comme mystère, ni l'incarnation comme paradigme. En ce sens, une fois atteinte leur plénitude au cours des années vingt et trente, elles fournissent à sa pensée un socle immuable. Mais l'évolution historique l'oblige à faire subir à son paradigme des inflexions auxquelles il ne se résigne que dans le tourment et la nostalgie. J'ajouterais que, 
si l'on prend le terme dans sa radicalité, Groulx n'est pas un moderne et toute la distance du théocentrisme le sépare des penseurs allemands comme Herder.

L'auteur me reproche de ramener au romantisme la croyance de Groulx (p. 126) ${ }^{27}$. Me suis-je mal exprimé ? m'at-il mal compris ? En tout cas, il ne tient pas un compte suffisant de la psychologie de Groulx et oublie que je parle du Groulx de la jeunesse, non pas de la maturité. En revanche, il a parfaitement raison de relever que, discutant de sa formation ecclésiastique, j’aurais dû citer le passage de son journal du 6 janvier 1902 où Groulx indique que « la théologie prend les plus nombreuses de [s]es heures [d']études». Pour sa part, l'auteur aurait pu reproduire cet extrait provenant de la même source, un paragraphe plus bas : "Ma vieille passion pour la lecture $[\ldots]$ m'amène trop souvent à faire du rabais sur l'étude de la théologie. » Je rappelle ces petites divergences pour attirer l'attention sur ce qui me semble une exagération de la science de Groulx en matière de thomisme avant 1909. Je suis d'autant plus à l'aise pour lui adresser cette amicale remontrance que cette critique ne menace nullement la solidité de la preuve à l'appui de sa thèse. L'auteur a démontré de façon incontestable que Groulx a pris très tôt le pli thomiste, qu'il vouait une admiration foncière au Docteur angélique et qu'il a parfait la connaissance de sa doctrine à Rome. Cela suffit, d'autant que, comme le rappelle l'auteur, Groulx n'a cessé de lire dans les domaines de la théologie, de l'exégèse, de la spiritualité et de la philosophie. Groulx le reconnaissait, ses études ecclésiastiques ont été gâchées par la maladie et la dispersion. Mal préparé, il ne peut s'inscrire à l'université de son choix, la Propagande, devant se contenter de la Minerve. En deux ans, il décroche le bonnet de docteur en philosophie (juin 1907) et en théologie (juin 1908). Ce n'était pas des doctorats de recherche, mais des diplômes sanctionnant une 
sorte de gavage scolastique. La seule thèse de doctorat que Groulx ait jamais écrite l'a été en histoire. Il ignorait l'histoire de la philosophie moderne et contemporaine. Ses études philosophiques l'avaient davantage déçu que ses études théologiques. S'il s'inscrit à l'université de Fribourg, c'est pour préparer une licence de lettres et non pour achever sa formation en philosophie en vue de l'enseignement de cette discipline (pp. 141, 152). Groulx n'a jamais été un professionnel de la théologie, encore moins de la philosophie. Cela n'empêche pas que l'outillage mental de Groulx, les catégories fondamentales de son activité intellectuelle et les orientations essentielles de sa pensée lui ont été fournis par le catholicisme et la scolastique, en particulier thomiste.

Un plan davantage chronologique aurait permis à l'auteur de montrer encore mieux qu'il ne l'a fait que la théologie de l'histoire de Groulx dans sa version développée et organique, qui lui permettait de saisir la vie nationale sur tous les plans et dans toutes ses dimensions, ne lui a pas été donnée toute faite, même s'il était le disciple de $\mathrm{M}^{\mathrm{gr}}$ Laflèche, de Tardivel, de $\mathrm{M}^{\mathrm{gt}}$ Pâquet, et un admirateur de Bourassa. Cela a plutôt été pour lui une conquête, qui n'excluait ni les approximations progressives, ni les reprises. Sa démarche d'ailleurs n'était pas solitaire. Dans la cathédrale Notre-Dame de Paris, le 13 juillet 1937, le cardinal Eugenio Pacelli, le futur Pie XII, prononçait un discours sur la vocation de la France, où l'on trouve jusqu'au vocabulaire de Groulx :

Mais ces aspirations mêmes que, malgré la grande variété de leurs manifestations, nous retrouvons à chaque génération française depuis les origines, comment les expliquer? Inutile d'invoquer je ne sais quel fatalisme ou quel déterminisme racial. À la France d'aujourd'hui, qui l'interroge, la France d'autrefois va répondre en donnant à cette hérédité son vrai nom: la vocation. Car, mes Frères, les peuples, comme les individus, ont 
leur vocation providentielle; comme les individus, ils sont prospères ou misérables, ils rayonnent ou demeurent obscurément stériles, selon qu'ils sont dociles ou rebelles à leur vocation.

Le temporel est une réalité mixte, où le spirituel et le profane se compénètrent à ce point que l'hérédité est spirituelle et que la fidélité à la vocation providentielle décide de la prospérité matérielle.

La thèse très systématique du professeur Cornett appelle un complément de nature à en atténuer la rigidité occasionnelle. Je crois que l'œuvre de Groulx n'est pas structurée par une seule logique, la philosophico-théologique, mais par une pluralité de logiques. Ces logiques sont fermement tenues en laisse par la logique religieuse, qui hiérarchise l'ensemble en toute souveraineté et interdit aux logiques subordonnées de se dresser contre la primauté du spirituel, en définitive contre l'ordre providentiel. Groulx était un traditionaliste et un catholique : l'ordre ne peut être pour lui qu'hétéronome. Mais la subordination, en droit comme en fait, de la logique politique et de la logique économique, par exemple, au catholicisme comme doctrine et comme vision du monde ne les empêche pas d'obéir, dans une certaine mesure, à leur raison interne. Il me semble que Bock et Boily l'ont démontré de façon convaincante pour ce qui est du politique. Une comparaison me fera mieux comprendre. Dans l'ecclésiologie et le droit public catholiques, l'Église et l'État sont présentés comme des sociétés parfaites de même que les pouvoirs spirituel et temporel sont définis comme à la fois distincts mais non séparés, autonomes et pourtant hiérarchisés de sorte qu'en matière doctrinale et dans les questions mixtes le primat du spirituel doit être loyalement accepté. Ces subtilités rendent assez fidèlement les règles qui régissent les rapports entre les logiques groulxiennes. Admettre ces règles et observer leur 
fonctionnement, leur respect comme leur transgression qu'on n'a pas le droit d'exclure a priori - rendrait à l'activité intellectuelle de Groulx une part de son foisonnement, de ses pulsations, de ses polarités, de ses recherches, sans tomber dans une linéarité illusoire ou une dichotomie oublieuse des réalités intermédiaires.

Ce trop bref résumé n'est qu'une invitation à lire la savante thèse du professeur Cornett, où abondent les aperçus lumineux et les mises au point opportunes, telle celle sur la convergence du racisme nazi et du groulxisme par l'entremise de Gonzague de Reynold et de Gobineau. Cette légende concoctée par Mason Wade, faussaire à ses heures, a été relayée, inconsciemment sans doute, par Esther Delisle et Catherine Pomeyrols $^{28}$. L'analyse de la théologie et de la philosophie groulxiennes de l'histoire, par lesquelles le penseur construisait le sens de son existence et le sens du destin canadienfrançais, non seulement réduit la part du racisme et de l'antisémitisme dans le groulxisme, mais encore révèle à quel point ce dernier est ordonné par la primauté du spirituel.

Le professeur Cornett ne nie pas la complexité déroutante et les ambivalences de la pensée groulxienne. Parce qu'il en exhume une des logiques profondes et sans doute la plus importante, il parait convaincant quand il signale les tensions et même les contradictions (p. 288). On l'écoute. Car enfin, si tout est contradiction, il n'y a plus de contradictions. Cela tombe sous le sens. L'organicisme de Boily, la conceptualisation de la nation de Bock et l'incarnationisme de Cornett réduisent à néant les hyperboles bouchardiennes, que nous estimerons à peine plus que celles d'Esther Delisle. On évitera d'ailleurs de confondre l'adéquation d'une idéologie à son temps et à son milieu avec sa cohérence interne, et sa cohérence interne avec le succès qu'elle remporte ou non. 
Refermant la belle étude de Norman F. Cornett et gardant vive l'image d'un Groulx tendu, par conviction et par volonté, vers les réalités les plus hautes, me revenait à la mémoire l'invective de Mordecai Richler: «this vile little cleric was also a fascist ${ }^{29} \%$. J'aime à croire que ce crachat aura emprunté une trajectoire verticale et que la gravité aura bien servi la justice.

\section{NOTES}

${ }^{1}$ Ces citations sont de Patrice de La Tour du Pin (1911-1975), un des très grands poètes du dernier siècle, un poète de la spiritualité, un théopoète. La première est tirée de Petit Théâtre crépusculaire, Paris, Gallimard, 1963, p. 23 ; la seconde, de Une somme de poésie, tome I, Le jeu de l'bomme en lui-même, Paris, Gallimard, 1981, p. 332.

${ }^{2}$ On doit à ces trois travailleurs infatigables l'édition critique du Journal de Lionel Groulx (1895-1911), Montréal, Presses de l'Université de Montréal, 1984, 2 vol., ainsi que de sa Correspondance (1894-1967), dont les trois premiers tomes (1894-1915) ont paru avec le concours de Pierre Trépanier, Montréal, Fides, 1989, 1993 et 2003.

${ }^{3}$ Stéphane Pigeon, Lionel Groulx, critique de la Révolution tranquille (1956-1967), mémoire de M.A. (histoire), Université de Montréal, 1999, vi-119 p. ; Dominique Foisy-Geoffroy, Esdras Minville, le nationalisme économique et le catholicisme social, 1923-1939, mémoire de M.A. (histoire), Université de Montréal, 2001, x-216 p. ; Yves Bégin, Raison et sentiment : nationalisme et antinationalisme dans le Québec des années trente, mémoire de M.A. (histoire), Université de Montréal, 2001, vi-144 p.

${ }^{4}$ Xavier Gélinas, La droite intellectuelle québécoise et la Révolution tranquille (19561966), thèse de Ph. D. (histoire), York University, 2001, xii-656 p. Cette étude paraitra sous peu aux Presses de l'Université Laval.

${ }^{5}$ Pascale Ryan, Des intellectuels dans la Cité. La Ligue d'action nationale, $1933-$ 1960, Université du Québec à Montréal, Thèse de Ph. D. (histoire), 2001, x$311 \mathrm{p}$.

${ }^{6}$ Marie-Pier Luneau, Lionel Groulx. Le mythe du berger, Montréal, Leméac, 2003, 226 p. - Les nombres entre parenthèses dans le texte renvoient à l'œuvre dont il est question. 
${ }^{7}$ Damien-Claude Bélanger, Lionel Groulx et la Franco-Américanie, mémoire de M.A. (histoire), Université de Montréal, 2000, vii-184 p.

${ }^{8}$ Harold Bérubé, « La ville au cœur de la nation : l'utilisation du passé dans l'élaboration de l'identité urbaine ", Urban History Review / Revue d'bistoire urbaine, vol. 30, $\mathrm{n}^{\circ} 2$ (mars 2002), pp. 16-27.

${ }^{9}$ Michel Bock, Lionel Groulx, les minorités françaises et la construction de l'identité canadienne-française. Étude d'bistoire intellectuelle, Université d'Ottawa, thèse de Ph. D. (histoire), 2002, ix-401 p.

${ }^{10}$ Jules Michelet, Introduction à l'bistoire universelle, Préface, CEuvres complètes de J. Michelet, Paris, Flammarion, 1897, vol. 35, p. 446.

${ }^{11}$ Frédéric Boily, La pensée nationaliste de Lionel Groulx, Sillery, Septentrion, 2003, 229 p.

${ }^{12}$ Gérard Bouchard, Mistouk, Montréal, Boréal, 2002, 509 p.

${ }^{13}$ Gérard Bouchard, La nation québécoise au futur et au passé, Montréal, VLB, $1999,157 \mathrm{p}$.

${ }^{14}$ Gérard Bouchard, Les deux chanoines. Contradiction et ambivalence dans la pensée de Lionel Groulx, Montréal, Boréal, 2003, 313 p.

${ }^{15}$ Gilles Routhier et Jean-Philippe Warren, dir., Les visages de la foi. Figures marquantes du catholicisme québécois, Montréal, Fides, 2003, 371 p., voir pp. 161 177, «Un chevalier du catholicisme : Lionel Groulx (1878-1967) » par Frédéric Boily.

${ }^{16}$ Nive Voisine, dir., Histoire du catholicisme québécois, vol. 3, Jean Hamelin et Nicole Gagnon, Le XX ${ }^{e}$ siècle, tome I, 1898-1940, Montréal, Boréal Express, 1984, p. 386.

${ }^{17}$ Jean-Paul II, dir.], Catéchisme de l'Église catholique, Paris, Mame-Plon, 1992, $676 \mathrm{p}$.

${ }^{18}$ Lionel Groulx, Pourquoi nous sommes divisés. Une réponse du chanoine Lionel Groulx, Montréal, Éditions de l'Action nationale, 1943, 30 p. Imprimatur de Philippe Perrier, vicaire général.

${ }^{19}$ Arthur Maheux, Pourquoi sommes-nous divisés ?, Montréal, Beauchemin et Radio-Canada, 1943, 217 p. Avec l'approbation de l'Ordinaire.

${ }^{20}$ Mordecai Richler, Ob Canada! Ob Quebec! Requiem for a Divided Country, New York, Viking Penguin, 1992, p. 106.

${ }^{21}$ Lionel Groulx, Chemins de l'avenir, Montréal, Fides, 1964, 161 p. 
${ }^{22}$ Sylvie Beaudreau, «Déconstruire le rêve de nation. Lionel Groulx et la Révolution tranquille ", Revue d'histoire de l'Amérique française, vol. 56, n' 1 (été 2002), pp. 29-61; Pierre Trépanier, «Lionel Groulx, historien », Les Cabiers des Dix, $\mathrm{n}^{\circ} 47$ (1992), pp. 247-277. Plus récemment, j’ai publié deux autres articles sur Groulx : « Un projet d'enquête sur la jeunesse universitaire (1913) », Les Cabiers des Dix, no 54 (2000), pp. 137-169 ; «L'étudiant idéal vers 1913 », Les Cabiers des Dix, $\mathrm{n}^{\circ} 55$ (2001), pp. 117-148.

${ }^{23}$ Norman F. Cornett, The Role of Religion in Lionel Groulx's Nationalist Thought, thèse de Ph. D. (études religieuses), Université McGill, 2002, v-430 p.

${ }^{24}$ Pierre Trépanier, «Les tribulations d'André Dagenais », Les Cabiers des Dix, $\mathrm{n}^{\circ} 56$ (2002), pp. 235-295; « Le philosophe André Dagenais devant la critique », Les Cabiers des Dix, n 57 (2003), pp. 205-262.

${ }^{25}$ Pierre Trépanier, « Notes pour une histoire des droites intellectuelles canadiennes-françaises à travers leurs principaux représentants (1770-1970) », Les Cabiers des Dix, no 48 (1993), pp. 119-164.

${ }^{26}$ Cité par Pierre Trépanier, "L'histoire intellectuelle selon Jean de Viguerie », Mens. Revue d'bistoire intellectuelle de l'Amérique française, vol. 2, $\mathrm{n}^{\circ} 1$ (automne 2001), pp. 5-15, voir p. 5.

${ }^{27}$ Pierre Trépanier, « Ascèse et action. Les impatiences de Lionel Groulx (18991906). Introduction II », dans Lionel Groulx, Correspondance, tome I, p. lxxix.

${ }^{28}$ Esther Delisle, Le Traître et le Juif. Lionel Groulx, Le Devoir, et le délire du nationalisme d'extrême droite dans la province de Québec, 1929-1939, Outremont, L'Étincelle, 1992, 284 p. ; Catherine Pomeyrols, Les intellectuels québécois : formation et engagements (1919-1939), Paris et Montréal, L'Harmattan, 1996, 537 p.

${ }^{29}$ Mordecai Richler, op. cit., p. 89. 